\title{
Joins of DGA modules and sectional category
}

\author{
LuCía FERnÁNDEZ SuÁREZ \\ PIERRE GHIENNE \\ THOMAS KAHL \\ LuCILE VANDEMBROUCQ
}

\begin{abstract}
We construct an explicit semifree model for the fiber join of two fibrations $p: E \rightarrow B$ and $p^{\prime}: E^{\prime} \rightarrow B$ from semifree models of $p$ and $p^{\prime}$. Using this model, we introduce a lower bound of the sectional category of a fibration $p$ which can be calculated from any Sullivan model of $p$ and which is closer to the sectional category of $p$ than the classical cohomological lower bound given by the nilpotency of the kernel of $p^{*}: H^{*}(B ; \mathbb{Q}) \rightarrow H^{*}(E ; \mathbb{Q})$. In the special case of the evaluation fibration $X^{I} \rightarrow X \times X$ we obtain a computable lower bound of Farber's topological complexity $\operatorname{TC}(X)$. We show that the difference between this lower bound and the classical cohomological lower bound can be arbitrarily large.
\end{abstract}

55M30; 55P62

\section{Introduction}

The sectional category of a fibration $p: E \rightarrow B$, denoted by secat $p$, is the least integer $n$ such that the base space $B$ can be covered by $n+1$ open subspaces on each of which $p$ admits a section. If no such $n$ exists one sets secat $p=\infty$. This homotopy invariant of a fibration has been introduced by A S Schwarz [15] in the late 1950 's as a generalization of the Lusternik-Schnirelmann category of a space. The Lusternik-Schnirelmann category of a space $X$, cat $X$, is the least integer $n$ such that $X$ can be covered by $n+1$ open subspaces each of which is contractible in $X$ (if no such $n$ exists one sets cat $X=\infty$ ). If $X$ is a path-connected space with base point $x_{0}$ and $P X$ is the space of paths beginning at $x_{0}$ then cat $X$ is precisely the sectional category of the evaluation fibration $e v_{1}: P X \rightarrow X, \omega \mapsto \omega(1)$. References on Lusternik-Schnirelmann category and sectional category are Schwarz [15], James [12; 13] and Cornea-Lupton-Oprea-Tanré [2].

The concept of sectional category has been used to introduce measures for the complexity of certain problems. S Smale [16] (see also [2, sec. 9.4]) obtained results on the complexity of the root-finding problem for algebraic equations in terms 
of sectional category. Recently, M Farber [3; 4] defined the topological complexity of a space $X, \operatorname{TC}(X)$, to be the sectional category of the evaluation fibration $e v_{0,1}: X^{I} \rightarrow X \times X, \omega \mapsto(\omega(0), \omega(1))$. This notion of topological complexity plays an important role in the study of the motion planning problem in robotics.

In spite of the simplicity of the definition, it is very hard to calculate the sectional category of a fibration $p: E \rightarrow B$ and therefore one will usually have to accept to work with approximations. For a surjective fibration one easily shows that secat $p \leq$ cat $B$. Hence all upper bounds of cat $B$, such as the dimension of $B$ or its cone-length, are upper bounds of secat $p$ as well. A classical cohomological lower bound of secat $p$ is nil ker $p^{*}$, the nilpotency of the kernel of $p^{*}: H^{*}(B) \rightarrow H^{*}(E)$ (with respect to any coefficient ring), i.e. the least integer $n$ such that any $(n+1)$-fold cup product in ker $p^{*}$ is trivial (cf. [2, Section 9.3]). There are, of course, examples where nil ker $p^{*}=$ secat $p$ but in general the inequality nil ker $p^{*} \leq$ secat $p$ is strict. As is showing the case of Lusternik-Schnirelmann category, that is, the special case where $p$ is the evaluation fibration $e v_{1}: P X \rightarrow X$, the difference between the two numbers may actually be infinite.

A far better lower bound of secat $p$ than nil ker $p^{*}$ (at least when the coefficient ring is $\mathbb{Q}$ ) is the rational sectional category $\operatorname{secat}_{0} p$, i.e. the sectional category of a rationalization of $p$. In her thesis [5], A Fassò Velenik gave a characterization of secat $_{0} p$ in terms of a Sullivan model of $p$. Unfortunately, concrete computations based on this characterization turn out to be rather difficult due to the complexity of the algebraic manipulations involved. In the present article we introduce an approximation of secat $p$ which is not as good as $\operatorname{secat}_{0} p$ in general but much easier to calculate. This approximation, which we denote by Msecat $p$, is still a much better lower bound of secat $p$ than nil ker $p^{*}$, if we consider coefficients in $\mathbb{Q}$. Let us note here that we work over the field $\mathbb{Q}$ in the algebraic part of this article and that all spaces we consider are compactly generated Hausdorff spaces.

There is a classical equivalent definition of sectional category in terms of joins which is more appropriate for our purpose than the original one. Denote by $*_{B}^{n} E$ the $n-$ fold fiber join of the fibration $p: E \rightarrow B$ and by $j^{n} p: *_{B}^{n} E \rightarrow B$ the $n$th join map. If $B$ is normal then secat $p \leq n$ if and only if $j^{n} p$ has a section. We recall this fact and the join construction in section 2. Let $A_{P L}$ denote Sullivan's functor of polynomial forms from spaces to commutative cochain algebras. Consider the morphism $A_{P L}\left(j^{n} p\right): A_{P L}(B) \rightarrow A_{P L}\left(*_{B}^{n} E\right)$ as a morphism of $A_{P L}(B)$-modules. In section 5, we define the invariant Msecat $p$ to be the least integer $n$ for which $A_{P L}\left(j^{n} p\right)=\phi \circ i$ where $\phi$ is a quasi-isomorphism of $A_{P L}(B)$-modules and $i$ is a morphism of $A_{P L}(B)$-modules which admits a retraction of $A_{P L}(B)$-modules. We show that nil ker $p^{*} \leq$ Msecat $p \leq$ secat $p$ for fibrations with a normal base space (cf. 
Theorem 5.2). In the special case of the evaluation fibration $e v_{1}: P X \rightarrow X$ of a simply connected space of finite type, Msecat coincides with the well-known invariant Mcat $X$ (cf. Proposition 5.6) which in turn is known to be the rational category of $X$ (cf. Hess [11]). The invariant Msecat generalizes the invariant Mcat hence in the same way as secat generalizes cat. The fact that Mcat is rational category does, however, not generalize to Msecat and Msecat does not in general equal rational sectional category.

The computability of the invariant Msecat relies on an algebraic join construction which we develop in sections 3 and 4 . Let $(A, d)$ be a commutative cochain algebra. In section 3, we define the join $(M, d) *_{(A, d)}(N, d)$ of two $(A, d)$-semifree extensions $(M, d)$ and $(N, d)$ of $(A, d)$. This is an explicitly defined semifree extension of $(A, d)$. Moreover, if $(M, d)$ and $(N, d)$ are minimal semifree $(A, d)$-modules, so is $(M, d) *_{(A, d)}(N, d)$. Consider two fibrations $p: E \rightarrow B$ and $p^{\prime}: E^{\prime} \rightarrow B$ between simply connected spaces of finite type and suppose that $\alpha:(A, d) \stackrel{\sim}{\longrightarrow} A_{P L}(B)$ is a commutative cochain algebra model of the base space $B$ and that $(M, d)$ and $(N, d)$ are semifree extensions of $(A, d)$ such that there exist quasi-isomorphisms of $(A, d)-$ modules $(M, d) \stackrel{\sim}{\longrightarrow} A_{P L}(E)$ and $(N, d) \stackrel{\sim}{\longrightarrow} A_{P L}\left(E^{\prime}\right)$ which extend $A_{P L}(p) \circ \alpha$ and $A_{P L}\left(p^{\prime}\right) \circ \alpha$. We establish in section 4 that the inclusion $(A, d) \rightarrow(M, d) *(A, d)(N, d)$ is an $(A, d)$-module model of the topological join map $E *_{B} E^{\prime} \rightarrow B$. Iterating the join construction, we define the $n$-fold join $*_{(A, d)}^{n}(M, d)$ of $(M, d)$ and obtain an explicit $(A, d)$-module model of the $n$th join map $j^{n} p: *_{B}^{n} E \rightarrow B$. The number Msecat $p$ is then the least $n$ such that the inclusion $(A, d) \rightarrow *_{(A, d)}^{n}(M, d)$ admits a retraction of $(A, d)$-modules (cf. Theorem 5.4). Through this result one obtains an effective method to compute the invariant Msecat $p$ from a Sullivan model of $p$.

As an example we consider Farber's topological complexity TC. Let $X$ be a simply connected space of finite type with Sullivan model $(\Lambda V, d)$. There is a well-known explicit minimal model of the evaluation fibration $e v_{0,1}: X^{I} \rightarrow X \times X$ which can be determined from $(\Lambda V, d)$. This model and the algebraic join construction permit one to calculate the invariant $\operatorname{MTC}(X)=\operatorname{Msecat} e v_{0,1}$ which is a lower bound of $\operatorname{TC}(X)$. Note that since $e v_{0,1}$ is the mapping path fibration associated to the diagonal map $X \rightarrow X \times X, e v_{0,1}^{*}$ can be identified with the cup product $\cup: H^{*}(X) \otimes H^{*}(X) \rightarrow$ $H^{*}(X)$. If $X$ is a formal space, i.e. a space whose rational homotopy type is entirely determined by its cohomology algebra, one has $\operatorname{MTC}(X)=$ nil ker $\cup$. But already for the simplest example of a non-formal space, one calculates that $\operatorname{MTC}(X)=3$ and nil ker $\cup=2$. We show finally that the difference between the two lower bounds is unbounded. 


\section{Sectional category and joins}

Recall from the introduction that the category of spaces in which we shall work throughout this article is the category of compactly generated Hausdorff spaces. All categorical constructions (products, pullbacks etc.) are carried out in this category.

In this section we recall the link between joins and the sectional category mentioned in the introduction.

Definition 2.1 The (fiber) join of two maps $p: E \rightarrow B$ and $p^{\prime}: E^{\prime} \rightarrow B$, denoted by $E *_{B} E^{\prime}$, is the double mapping cylinder of the projections $E \times{ }_{B} E^{\prime} \rightarrow E$ and $E \times_{B} E^{\prime} \rightarrow E^{\prime}$, i.e. the quotient space $\left(\left(E \times_{B} E^{\prime}\right) \times I \amalg E \amalg E^{\prime}\right) / \sim$ where $\left(e, e^{\prime}, 0\right) \sim e$, $\left(e, e^{\prime}, 1\right) \sim e^{\prime}$. The join map of $p$ and $p^{\prime}$ is the map $j_{p, p^{\prime}}: E *_{B} E^{\prime} \rightarrow B$ defined by $j_{p, p^{\prime}}\left(\left[e, e^{\prime}, t\right]\right)=p(e)=p^{\prime}\left(e^{\prime}\right), j_{p, p^{\prime}}([e])=p(e)$, and $j_{p, p^{\prime}}\left(\left[e^{\prime}\right]\right)=p^{\prime}\left(e^{\prime}\right)$. The $n$-fold join and the $n$th join map of $p$ are iteratively defined by $*_{B}^{0} E=E, *_{B}^{n} E=$ $\left(*_{B}^{n-1} E\right) *_{B} E, j^{0} p=p$, and $j^{n} p=j_{j^{n-1} p, p}$.

Theorem 2.2 Let $p: E \rightarrow B$ be a fibration. If $B$ is normal then secat $p \leq n$ if and only if $j^{n} p$ has a section.

Proof The result is well-known, at least when $B$ is paracompact (cf. James [12]). We include a short proof for the convenience of the reader.

Suppose first that secat $p \leq n$. We show by induction that for each $0 \leq m \leq n$ there exists an open cover $U_{0}, \ldots, U_{n-m}$ of $B$ such that $j^{m} p$ has a section on $U_{0}$ and $p$ has a section on each of the remaining $U_{i}$. For $m=0$ this is just the hypothesis that secat $p \leq n$. Suppose that the assertion holds for $0 \leq m<n$. Then there exists an open cover $U_{0}, \ldots, U_{n-m}$ of $B$, a section $\sigma_{0}: U_{0} \rightarrow *_{B}^{m} E$ of $j^{m} p$, and sections $\sigma_{i}: U_{i} \rightarrow E$ of $p(1 \leq i \leq n-m)$. Since $B$ is normal, there exist open covers $V_{0}, \ldots, V_{n-m}$ and $W_{0}, \ldots, W_{n-m}$ of $B$ such that $\bar{V}_{i} \subset W_{i} \subset \bar{W}_{i} \subset U_{i}$. Set $A_{0}=\bar{V}_{0} \cap\left(B \backslash W_{1}\right)$, $A_{1}=\bar{V}_{1} \cap\left(B \backslash W_{0}\right)$, and $A_{2}=\bar{W}_{0} \cap \bar{W}_{1} \cap\left(\bar{V}_{0} \cup \bar{V}_{1}\right)$. Then $A_{0}, A_{1}$, and $A_{2}$ are closed subspaces of $B, A_{0} \cup A_{1} \cup A_{2}=\bar{V}_{0} \cup \bar{V}_{1}$, and $A_{0} \cap A_{1}=\varnothing$. Since $B$ is normal, by Urysohn's Lemma, there exists a continuous map $\phi: B \rightarrow I$ such that $\phi\left(A_{0}\right) \subset\{0\}$ and $\phi\left(A_{1}\right) \subset\{1\}$. Define a section $\sigma$ of $j^{m+1} p$ on $\bar{V}_{0} \cup \bar{V}_{1}$ by

$$
\sigma(x)= \begin{cases}{\left[\sigma_{0}(x)\right],} & x \in A_{0}, \\ {\left[\sigma_{1}(x)\right],} & x \in A_{1}, \\ {\left[\sigma_{0}(x), \sigma_{1}(x), \phi(x)\right],} & x \in A_{2} .\end{cases}
$$

Consider the open cover $O_{0}, \ldots O_{n-m-1}$ of $B$ given by $O_{0}=V_{0} \cup V_{1}$ and $O_{i}=U_{i+1}$, $i=1, \ldots, n-m-1$. On $O_{0}, \sigma$ is a section of $j^{m+1} p$. On each of the remaining $O_{i}$, $p$ has a section by hypothesis. This terminates the inductive step. 
Suppose now that $j^{n} p$ has a section $s: B \rightarrow *_{B}^{n} E$. By Lemma 2.4 below, $*_{B}^{n} E$ can be covered by $n+1$ open subspaces $U_{0}, \ldots, U_{n}$ on each of which the projection $\bar{p}_{n}:\left(*_{B}^{n} E\right) \times_{B} E \rightarrow *_{B}^{n} E$ has a section. The inverse images $s^{-1}\left(U_{i}\right)$ form a cover of $B$ by open subspaces on each of which $p$ has a section. Therefore secat $p \leq n$.

Remark 2.3 If $p: E \rightarrow B$ and $p^{\prime}: E^{\prime} \rightarrow B$ are fibrations, so is the join map $j_{p, p^{\prime}}: E *_{B} E^{\prime} \rightarrow B$. Indeed, if $\lambda: E \times_{B} B^{I} \rightarrow E^{I}$ and $\lambda^{\prime}: E^{\prime} \times_{B} B^{I} \rightarrow E^{\prime I}$ are lifting maps for $p$ and $p^{\prime}$ then a lifting map $\phi:\left(E *_{B} E^{\prime}\right) \times_{B} B^{I} \rightarrow\left(E *_{B} E^{\prime}\right)^{I}$ for $j_{p, p^{\prime}}$ is given by $\phi\left(\left[e, e^{\prime}, t\right], \omega\right)(s)=\left[\lambda(e, \omega)(s), \lambda^{\prime}\left(e^{\prime}, \omega\right)(s), t\right], \phi([e], \omega)(s)=[\lambda(e, \omega)(s)]$, and $\phi\left(\left[e^{\prime}\right], \omega\right)(s)=\left[\lambda^{\prime}\left(e^{\prime}, \omega\right)(s)\right]$. Note that $\phi$ is continuous since we are working with compactly generated spaces. It follows, by induction, that the $n$th join map of a fibration is again a fibration and hence that it has a section if and only if it has a homotopy section.

In the proof of Theorem 2.2 we used the following lemma. We shall need this lemma again in the proof of the inequality Msecat $p \geq$ nil ker $p^{*}$ (cf. Theorem 5.2).

Lemma 2.4 Consider a fibration $p: E \rightarrow B$ and form the pullback diagram:

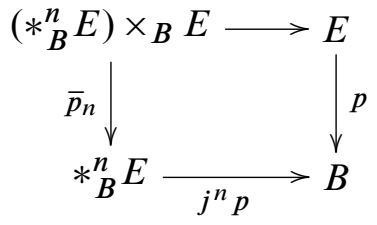

Then secat $\bar{p}_{n} \leq n$.

Proof We proceed by induction. For $n=0$, the map $E \rightarrow E \times_{B} E, e \mapsto(e, e)$ is a section of $\bar{p}_{n}$. Suppose that $n>0$ and that the assertion holds for $n-1$. The spaces $E$ and $*_{B}^{n-1} E$ are embedded as closed subspaces in $*_{B}^{n} E$ and there are canonical projections $\pi: *_{B}^{n} E \backslash *_{B}^{n-1} E \rightarrow E$ and $\tilde{\pi}: *_{B}^{n} E \backslash E \rightarrow *_{B}^{n-1} E$. Let $U_{0}$ be the open subspace $*_{B}^{n} E \backslash *{ }_{B}^{n-1} E$ of $*_{B}^{n} E$. We have $\left.j^{n} p\right|_{U_{0}}=p \pi$. The inductive hypothesis implies that $*_{B}^{n-1} E$ can be covered by $n$ open subspaces $V_{1}, \ldots, V_{n}$ such that each restriction of the join map $\left.j^{n-1} p\right|_{V_{i}}: V_{i} \rightarrow B$ factors through $p$. Consider the open subspaces $U_{i}=\tilde{\pi}^{-1}\left(V_{i}\right)$ of $*_{B}^{n} E$. The $n+1$ open subspaces $U_{0}, U_{1} \ldots, U_{n}$ of $*_{B}^{n} E$ cover $*_{B}^{n} E$. The restriction of the join map $j^{n} p$ to any of these open subspaces factors through $p$. Therefore the projection $\bar{p}_{n}:\left(*_{B}^{n} E\right) \times_{B} E \rightarrow *_{B}^{n} E$ has a section on each $U_{i}$. This shows that secat $\bar{p}_{n} \leq n$. 


\section{Joins of semifree modules}

The purpose of this section is to define joins of semifree extensions of a commutative cochain algebra. Recall that we are working over $\mathbb{Q}$. All graded vector spaces we consider will be $\mathbb{Z}$-graded with upper degree and all differential vector spaces will be cochain complexes, i.e. the differential raises the upper degree by one. The $n t h$ suspension $s^{-n} V$ of a graded vector space $V$ is defined by $\left(s^{-n} V\right)^{i}=V^{i-n}$.

Definition 3.1 Let $(A, d)$ be a differential algebra. A semifree extension of an $(A, d)-$ module $(M, d)$ is an $(A, d)$-module of the form $(M \oplus A \otimes X, d)$ where the action is the one of the direct sum, the differential on $M$ is the differential of $(M, d)$, and $X$ admits a direct sum decomposition $X=\bigoplus_{i=0}^{\infty} X_{i}$ such that $d\left(X_{0}\right) \subset M$ and $d\left(X_{n}\right) \subset M \oplus A \otimes\left(\bigoplus_{i=0}^{n-1} X_{i}\right)$ for $n \geq 1$. A semifree $(A, d)$-module is a semifree extension of the trivial $(A, d)$-module 0 .

For the remainder of this section we fix a commutative cochain algebra $(A, d)$ and two semifree extensions $(M, d)=(A \oplus A \otimes X, d)$ and $(N, d)=(A \oplus A \otimes Y, d)$ of $(A, d)$ We define the join $(M, d) *(A, d)(N, d)$ of $(M, d)$ and $(N, d)$ which will again be a semifree extension of $(A, d)$. Forgetting the differential, $(M, d) *_{(A, d)}(N, d)$ is the free graded $A$-module $A \oplus A \otimes S^{-1} X \otimes Y$. In order to define the differential of $(M, d) *{ }_{(A, d)}(N, d)$, we decompose the differential in $(M, d)$ of an element $m \in M$ as

$$
d m=d_{0} m+d_{+} m
$$

where $d_{0} m \in A$ and $d_{+} m \in A \otimes X$. Using the same notation, we decompose the differential in $(N, d)$ of an element $n \in N$. Consider elements $x \in X$ and $y \in Y$ and write

$$
d_{+} x=\sum_{i} a_{i} \otimes x_{i}, \quad \text { and } \quad d_{+} y=\sum_{j} b_{j} \otimes y_{j} .
$$

The differential of the element $s^{-1} x \otimes y$ in $(M, d) *(A, d)(N, d)$ is then defined by

$$
\begin{aligned}
d\left(s^{-1} x \otimes y\right)= & (-1)^{|x|} d_{0} x d_{0} y+\sum_{i}(-1)^{\left|a_{i}\right|+1} a_{i} \otimes s^{-1} x_{i} \otimes y \\
& +\sum_{j}(-1)^{(|x|+1)\left(\left|b_{j}\right|+1\right)} b_{j} \otimes s^{-1} x \otimes y_{j} .
\end{aligned}
$$

We extend this differential to the whole join $(M, d) *_{(A, d)}(N, d)$ by setting

$$
d\left(a \otimes s^{-1} x \otimes y\right)=d a \otimes s^{-1} x \otimes y+(-1)^{|a|} a \cdot d\left(s^{-1} x \otimes y\right) .
$$


Proposition 3.2 below assures that $d$ is indeed an $(A, d)$-module differential in $(M, d) *_{(A, d)}(N, d)$. It is an easy exercise to check that $(M, d) *_{(A, d)}(N, d)$ is a semifree extension of $(A, d)$. Moreover, if $(A, d)$ is augmented and $(M, d)$ and $(N, d)$ are minimal semifree $(A, d)$-modules, i.e. the differentials in $\mathbb{Q} \otimes_{(A, d)}(M, d)$ and $\mathbb{Q} \otimes_{(A, d)}(N, d)$ are zero, then $(M, d) *_{(A, d)}(N, d)$ is also minimal.

Proposition $3.2 d^{2}\left(s^{-1} x \otimes y\right)=0$.

Proof Write $d_{+} x_{i}=\sum_{k} a_{i k} \otimes x_{i k}$ and $d_{+} y_{j}=\sum_{l} b_{j l} \otimes y_{j l}$. Since

$$
\begin{aligned}
0 & =d^{2} x \\
& =d\left(d_{0} x+\sum_{i} a_{i} \otimes x_{i}\right) \\
& =d d_{0} x+\sum_{i} d a_{i} \otimes x_{i}+\sum_{i}(-1)^{\left|a_{i}\right|} a_{i} d_{0} x_{i}+\sum_{i}(-1)^{\left|a_{i}\right|} a_{i} d_{+} x_{i},
\end{aligned}
$$

we have $d d_{0} x=-\sum_{i}(-1)^{\left|a_{i}\right|} a_{i} d_{0} x_{i}=\sum_{i}(-1)^{\left|a_{i}\right|+1} a_{i} d_{0} x_{i}$ and

$$
\sum_{i} d a_{i} \otimes x_{i}=-\sum_{i}(-1)^{\left|a_{i}\right|} a_{i} d_{+} x_{i}=\sum_{i, k}(-1)^{\left|a_{i}\right|+1} a_{i} a_{i k} \otimes x_{i k} .
$$

Similarly, $d d_{0} y=\sum_{j}(-1)^{\left|b_{j}\right|+1} b_{j} d_{0} y_{j}$ and

$$
\sum_{j} d b_{j} \otimes y_{j}=\sum_{j, l}(-1)^{\left|b_{j}\right|+1} b_{j} b_{j l} \otimes y_{j l} .
$$

Use $\sigma$ to denote the isomorphism

$$
A \otimes X \otimes Y \rightarrow A \otimes s^{-1} X \otimes Y, \quad a \otimes x \otimes y \mapsto(-1)^{|a|} a \otimes s^{-1} x \otimes y
$$

and $T$ to denote the isomorphism

$$
A \otimes X \rightarrow X \otimes A, \quad a \otimes x \mapsto(-1)^{|a||x|} x \otimes a .
$$

Applying $\sigma$ to the identity $\sum_{i} d a_{i} \otimes x_{i} \otimes y=\sum_{i, k}(-1)^{\left|a_{i}\right|+1} a_{i} a_{i k} \otimes x_{i k} \otimes y$, one obtains the identity

$$
\sum_{i}(-1)^{\left|a_{i}\right|+1} d a_{i} \otimes s^{-1} x_{i} \otimes y=\sum_{i, k}(-1)^{\left|a_{i k}\right|+1} a_{i} a_{i k} \otimes s^{-1} x_{i k} \otimes y .
$$

Applying $\sigma \circ\left(T \otimes i d_{Y}\right)$ to the identity

$$
\sum_{j} x \otimes d b_{j} \otimes y_{j}=\sum_{j, l}(-1)^{\left|b_{j}\right|+1} x \otimes b_{j} b_{j l} \otimes y_{j l},
$$

Algebraic 83 Geometric Topology, Volume 6 (2006) 
one obtains the identity

$$
\begin{aligned}
\sum_{j}(-1)^{|x|+\left|b_{j}\right|+|x|\left|b_{j}\right|} d b_{j} \otimes s^{-1} x \otimes y_{j} \\
=\sum_{j, l}(-1)^{|x|\left|b_{j}\right|+|x|\left|b_{j l}\right|+\left|b_{j l}\right|} b_{j} b_{j l} \otimes s^{-1} x \otimes y_{j l} .
\end{aligned}
$$

Using the different formulae above one easily verifies that $d^{2}\left(s^{-1} x \otimes y\right)=0$.

Definition 3.3 The $n$-fold join of $(M, d)$ is iteratively defined by $*_{(A, d)}^{0}(M, d)=$ $(M, d)$ and $*_{(A, d)}^{n}(M, d)=\left(*_{(A, d)}^{n-1}(M, d)\right) *_{(A, d)}(M, d)$.

Remarks 3.4 (i) Note that $*_{(A, d)}^{n}(M, d)$ is a semifree extension of $(A, d)$. Moreover, if $(M, d)$ is a minimal semifree $(A, d)$-module then $*_{(A, d)}^{n}(M, d)$ is a minimal semifree $(A, d)$-module as well.

(ii) We have

$$
*_{(A, d)}^{n}(M, d)=\left(A \oplus A \otimes s^{-n} X^{\otimes n+1}, d\right) .
$$

Consider elements $x_{0}, \ldots, x_{n} \in X$ and write $d_{+} x_{i}=\sum_{j_{i}} a_{i j_{i}} \otimes x_{i j_{i}}$. An easy induction shows that

$$
\begin{aligned}
& d\left(s^{-n} x_{0} \otimes \cdots \otimes x_{n}\right)=(-1)^{\sum_{k=1}^{n}\left(k\left|x_{n-k}\right|+k-1\right)} d_{0} x_{0} \cdots d_{0} x_{n} \\
& +\sum_{i=0}^{n} \sum_{j_{i}}(-1)^{\left(\left|a_{i j_{i}}\right|+1\right)\left(\left|x_{0}\right|+\cdots+\left|x_{i-1}\right|+n\right)} a_{i j_{i}} \otimes s^{-n} x_{0} \otimes \cdots \otimes x_{i j_{i}} \otimes \cdots \otimes x_{n} .
\end{aligned}
$$

(iii) Consider a morphism of commutative cochain algebras $(A, d) \rightarrow(B, d)$ and the $(B, d)$-semifree extension of $(B, d)$ defined by $(N, d)=(B, d) \otimes_{(A, d)}(M, d)$. The formula for the differential given in (ii) shows that

$$
*_{(B, d)}^{n}(N, d)=(B, d) \otimes_{(A, d)} *_{(A, d)}^{n}(M, d) .
$$

\section{Topological versus algebraic joins}

Our goal in this section is to show that the algebraic joins of the preceding section model topological joins. In this and the following sections we make frequent use of the homotopy theory of modules over a DGA and, in particular, of the following well-known result:

Algebraic $\&$ Geometric Topology, Volume 6 (2006) 
Theorem 4.1 Let $(A, d)$ be a differential algebra. The category of $(A, d)$-modules is a proper closed model category where weak equivalences are quasi-isomorphisms, fibrations are surjective morphisms, and cofibrations are morphims having the left lifting property with respect to surjective quasi-isomorphisms. A morphism is a cofibration if and only if it is a retract of the inclusion of a semifree extension.

We refer the reader to Goerss and Jardine [10] for the axioms of closed model categories. A closed model category is called proper if the class of weak equivalences is closed under base change along fibrations and cobase change along cofibrations. As is customary we denote weak equivalences by $\stackrel{\sim}{\longrightarrow}$, fibrations by $\rightarrow$, and cofibrations by $\succ$. For the convenience of the reader we include the following proof.

Proof of Theorem 4.1 We do not use the fact that we are working over $\mathbb{Q}$ and the proof works for an arbitrary commutative ground ring. We first show that inclusions of semifree extensions are cofibrations. Consider a semifree extension $(M \oplus A \otimes$ $\left.\left(\bigoplus_{i=0}^{\infty} X_{i}\right), d\right)$ of an $(A, d)$-module $(M, d)$ and a commutative diagram of $(A, d)$ modules

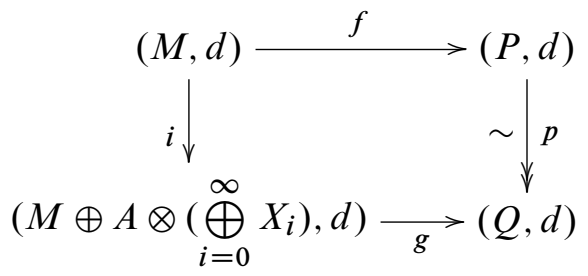

where $i$ is the inclusion. Suppose inductively that we have constructed a lifting $\lambda$ for the diagram up to $\left(M \oplus A \otimes\left(\bigoplus_{i=0}^{n} X_{i}\right), d\right)$. Let $\mathcal{B} \subset X_{n+1}$ be a basis and $x \in \mathcal{B}$. Then $\lambda(d x)$ is defined and $d \lambda(d x)=0$. Since $p$ is surjective, there exists an element $\xi \in P$ such that $p(\xi)=g(x)$. Then $\lambda(d x)-d \xi$ is a cocycle in ker $p$. Since $p$ is a surjective quasi-isomorphism, ker $p$ is acyclic and there exists an element $y \in \operatorname{ker} p$ such that $d y=\lambda(d x)-d \xi$. Set $\lambda(x)=\xi+y$. This defines $\lambda$ in $\left(M \oplus A \otimes\left(\bigoplus_{i=0}^{n+1} X_{i}\right), d\right)$. It follows that a lifting exists and hence that $i$ is a cofibration. Axiom CM1 (existence of finite limits and colimits) follows from the fact that the category of modules over the ground ring is complete and cocomplete. The fact that the quasi-isomorphisms have the " $2=3$ " property $(\mathrm{CM} 2)$ and are closed under retracts follows from the corresponding properties of isomorphisms. The fibrations are closed under retracts because surjective maps are closed under retracts. It is a general fact that any class of morphisms in a category which is defined by having the left lifting property with respect to another class of morphisms is closed under retracts. Therefore the class of cofibrations is closed 
under retracts and CM3 holds. We check the factorization axiom CM5. Consider a morphism $f:(M, d) \rightarrow(N, d)$ of $(A, d)$-modules. Consider the acyclic semifree $(A, d)$-module $\left(A \otimes\left(\tilde{N} \oplus s^{-1} \tilde{N}\right), \delta\right)$ where $\tilde{N}=\bigoplus_{n \in N} \mathbb{Q} \cdot n, \delta n=s^{-1} n$ and $\delta s^{-1} n=0$.

Then the inclusion $i:(M, d) \rightarrow(M, d) \oplus\left(A \otimes\left(\widetilde{N} \oplus s^{-1} \tilde{N}\right), \delta\right)$ is both a cofibration and a quasi-isomorphism. Let $p:(M, d) \oplus\left(A \otimes\left(\widetilde{N} \oplus s^{-1} \widetilde{N}\right), \delta\right) \rightarrow(N, d)$ be the morphism of $(A, d)$-modules defined by $p(m)=f(m), p(n)=n$, and $p\left(s^{-1} n\right)=d n$. Obviously, $p$ is surjective and $f=p \circ i$. This shows one part of CM5. In the proof of Félix-Halperin-Thomas, [8, 2.1(i)], it is shown that there is a factorization $f=p \circ i$ where $p$ is a surjective quasi-isomorphism and $i$ is the inclusion of a semifree extension. This shows the other part of CM5. We verify the lifting axiom CM4. One of the lifting properties is the definition of cofibrations. For the other one consider a commutative diagram of $(A, d)$-modules:

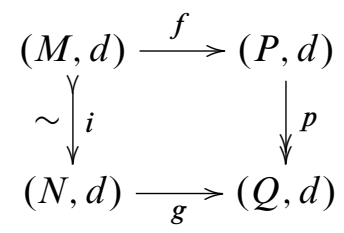

Form the pullback $(A, d)$-module $(N, d) \times_{(Q, d)}(P, d)$. Since $p$ is surjective, so is its base extension $\bar{p}:(N, d) \times_{(Q, d)}(P, d) \rightarrow(N, d)$. Choose a factorization of the canonical morphism $(i, f):(M, d) \rightarrow(N, d) \times(Q, d)(P, d)$ in a quasi-isomorphism $j:(M, d) \stackrel{\sim}{\longrightarrow}(R, d)$ and a surjective morphism $r:(R, d) \rightarrow(N, d) \times_{(Q, d)}(P, d)$. The composite $\bar{p} \circ r$ is a surjective quasi-isomorphism. Since $i$ is a cofibration, there exists a section $s$ of $\bar{p} \circ r$ such that $s \circ i=j$. Let $\bar{g}:(N, d) \times(Q, d)(P, d) \rightarrow(P, d)$ be the base extension of $g$. Then the composite $\bar{g} \circ r \circ s$ is a lifting for the above square. It follows that the category of $(A, d)$-modules is a closed model category.

We have seen that an inclusion of a semifree extension is a cofibration. By CM3, any retract of an inclusion of a semifree extension is a cofibration. Let $i$ be a cofibration and $i=p \circ j$ be a factorization such that $j$ is the inclusion of a semifree extension and $p$ is a surjective quasi-isomorphism. We have already mentioned that such a factorization exists. By CM4, there exists a section $s$ of $p$ such that $s \circ i=j$. This implies that $i$ is a retract of $j$. Thus a morphism is a cofibration if and only if it is a retract of the inclusion of a semifree extension. In particular, any cofibration is injective. Therefore we may use the 5-lemma to show that the cobase extension of a weak equivalence along a cofibration is a weak equivalence. Since, by definition, fibrations are surjetive, the 5-lemma implies that base extension of a weak equivalence along a fibration is a weak equivalence. It follows that the closed model category of $(A, d)$-modules is proper.

Algebraic ${ }^{6} \mathcal{G}$ Geometric Topology, Volume 6 (2006) 
Consider two fibrations $p: E \rightarrow B$ and $p^{\prime}: E^{\prime} \rightarrow B$ where $B, E$, and $E^{\prime}$ are simply connected spaces of finite type. Simply connected spaces are understood to be nonempty. A space is said to be of finite type if it has finite dimensional rational homology in every dimension. Let $A_{P L}$ be Sullivan's functor from spaces to commutative cochain algebras. Fix a commutative cochain algebra model $\alpha:(A, d) \stackrel{\sim}{\longrightarrow} A_{P L}(B)$. For any continuous map $f: S \rightarrow B$ the morphism of commutative cochain algebras $A_{P L}(f) \circ \alpha$ induces an $(A, d)$-module structure on $A_{P L}(S)$ such that $A_{P L}(f) \circ \alpha$ is a morphism of $(A, d)$-modules. Let $(M, d)=(A \oplus A \otimes X, d)$ and $(N, d)=(A \oplus A \otimes Y, d)$ be semifree extensions of $(A, d)$ such that there exist quasi-isomorphisms of $(A, d)$ modules $(M, d) \stackrel{\sim}{\longrightarrow} A_{P L}(E)$ and $(N, d) \stackrel{\sim}{\longrightarrow} A_{P L}\left(E^{\prime}\right)$ which extend $A_{P L}(p) \circ \alpha$ and $A_{P L}\left(p^{\prime}\right) \circ \alpha$. As in the preceding section we write $d=d_{0}+d_{+}$for the differentials of $(M, d)$ and $(N, d)$.

Theorem 4.2 (i) The morphism of $(A, d)$-modules $A_{P L}\left(j_{p, p^{\prime}}\right) \circ \alpha$ extends to a quasi-isomorphism of $(A, d)$-modules $(M, d) *_{(A, d)}(N, d) \stackrel{\sim}{\longrightarrow} A_{P L}\left(E *_{B} E^{\prime}\right)$.

(ii) The morphism of $(A, d)$-modules $A_{P L}\left(j^{n} p\right) \circ \alpha$ extends to a quasi-isomorphism of $(A, d)$-modules $*_{(A, d)}^{n}(M, d) \stackrel{\sim}{\longrightarrow} A_{P L}\left(*_{B}^{n} E\right)$.

Proof The second part follows from the first by induction. The proof of (i) is divided in 3 steps.

\section{Step 1: A model of the pullback}

Choose Sullivan models $\psi:(A \otimes \Lambda V, d) \stackrel{\sim}{\longrightarrow} A_{P L}(E)$ and $\psi^{\prime}:\left(A \otimes \Lambda V^{\prime}, d\right) \stackrel{\sim}{\longrightarrow}$ $A_{P L}\left(E^{\prime}\right)$ of $A_{P L}(p) \circ \alpha$ and $A_{P L}\left(p^{\prime}\right) \circ \alpha$. Since the inclusions $(A, d) \rightarrow(M, d)$ and $(A, d) \rightarrow(N, d)$ are cofibrations, by the lifting lemma (Baues [1, II.1.11]), there exist quasi-isomorphisms of $(A, d)$-modules $h:(M, d) \stackrel{\sim}{\longrightarrow}(A \otimes \Lambda V, d)$ and $h^{\prime}:(N, d) \stackrel{\sim}{\longrightarrow}\left(A \otimes \Lambda V^{\prime}, d\right)$ which extend the inclusions of $(A, d)$. Form the pushout of commutative cochain algebras:

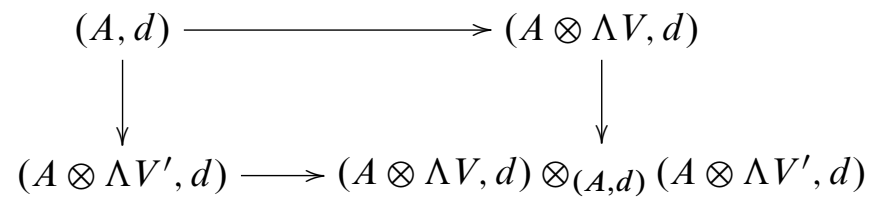

It follows from Félix-Halperin-Thomas $[9,15(\mathrm{c})]$ that the morphisms $A_{P L}\left(p r_{E}\right) \circ \psi$ and $A_{P L}\left(p r_{E}^{\prime}\right) \circ \psi^{\prime}$, where $p r_{E}: E \times_{B} E^{\prime} \rightarrow E$ and $p r_{E^{\prime}}: E \times_{B} E^{\prime} \rightarrow E^{\prime}$ are the projections, induce a quasi-isomorphism of commutative cochain algebras

$$
(A \otimes \Lambda V, d) \otimes_{(A, d)}\left(A \otimes \Lambda V^{\prime}, d\right) \rightarrow A_{P L}\left(E \times_{B} E^{\prime}\right) .
$$


By $[9,6.7]$, since $h$ and $h^{\prime}$ are quasi-isomorphisms between semifree $(A, d)$-modules, the morphism

$$
h \otimes_{A} h^{\prime}:(M, d) \otimes_{(A, d)}(N, d) \rightarrow(A \otimes \Lambda V, d) \otimes_{(A, d)}\left(A \otimes \Lambda V^{\prime}, d\right)
$$

is a quasi-isomorphism. Since $A$ is commutative, $(M, d) \otimes_{(A, d)}(N, d)$ is an $(A, d)-$ module and $h \otimes_{A} h^{\prime}$ a quasi-isomorphism of $(A, d)$-modules. Note that

$$
(M, d) \otimes_{(A, d)}(N, d)=\left(M \otimes_{A} N, d\right)=(A \oplus A \otimes(X \oplus Y \oplus X \otimes Y), d)
$$

contains both $(M, d)$ and $(N, d)$ as sub $(A, d)$-modules. Note also that if $d_{+} x=$ $\sum_{i} a_{i} \otimes x_{i}$ and $d_{+} y=\sum_{j} b_{j} \otimes y_{j}$ then the differential of $x \otimes y$ in $(M, d) \otimes_{(A, d)}(N, d)$ is given by

$$
\begin{aligned}
d(x \otimes y)= & d_{0} x \otimes y+\sum_{i} a_{i} \otimes x_{i} \otimes y+(-1)^{|x||y|} d_{0} y \otimes x \\
& +\sum_{j}(-1)^{|x|\left(\left|b_{j}\right|+1\right)} b_{j} \otimes x \otimes y_{j} .
\end{aligned}
$$

We have obtained the following commutative diagram of $(A, d)$-modules:

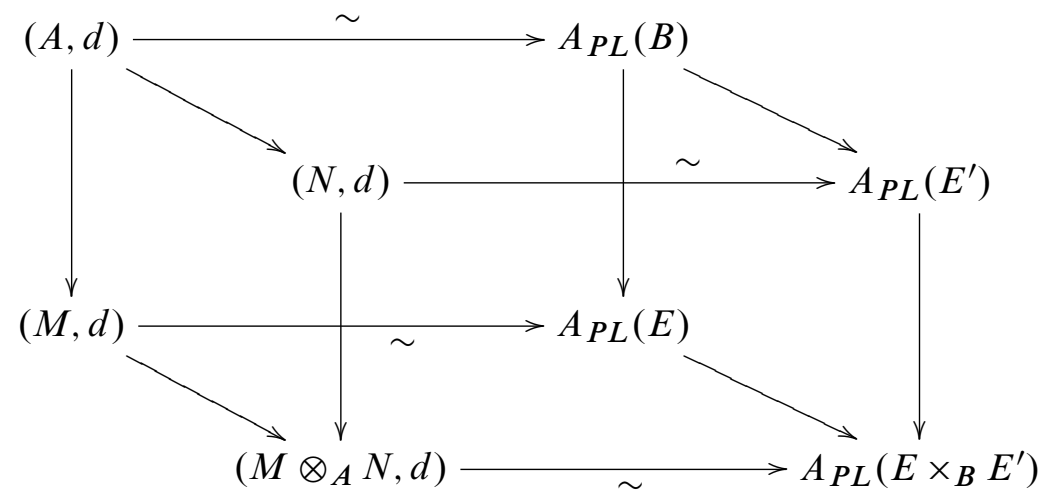

\section{Step 2: A model of the join map}

Consider the mapping cylinder factorization of the projection $p r_{E}^{\prime}: E \times_{B} E^{\prime} \rightarrow E^{\prime}$ in a cofibration $\iota: E \times{ }_{B} E^{\prime} \rightarrow Z$ and a homotopy equivalence $\rho: Z \rightarrow E^{\prime}$. We have the following pushout:

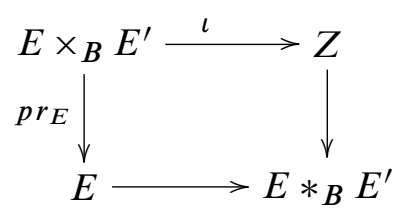

Algebraic $8 \mathcal{G}$ Geometric Topology, Volume 6 (2006) 
Let $v$ be the inclusion $(N, d) \rightarrow\left(M \otimes_{A} N, d\right)$. We construct the mapping path factorization of $v$. Consider the $(A, d)$-module

$$
(Q, D)=\left(M \otimes_{A} N \oplus N \oplus s^{-1} M \otimes_{A} N, D\right)
$$

where the action on $M \otimes_{A} N \oplus N$ is the one of the direct sum, $a \cdot s^{-1} w=$ $(-1)^{|a|} S^{-1} a w$, and the differential is given by

$$
\begin{aligned}
D\left(m \otimes_{A} n\right) & =d\left(m \otimes_{A} n\right)+s^{-1} m \otimes_{A} n \\
D n & =d n+s^{-1} v(n) \\
D s^{-1} w & =-s^{-1} d w .
\end{aligned}
$$

Let $i:(N, d) \rightarrow(Q, D)$ be the injection defined by $i(n)=v(n)-n$. One easily checks that this is a morphism of $(A, d)$-modules. We show that $i$ is both a cofibration and a quasi-isomorphism. Set $U=\mathbb{Q} \oplus X \oplus Y \oplus X \otimes Y$ where the elements of $\mathbb{Q}$ have degree 0 . Then $M \otimes_{A} N$ is the free graded $A$-module $A \otimes U$. Consider the acyclic semifree $(A, d)$-module $\left(A \otimes\left(U \oplus s^{-1} U\right), \delta\right)$ where $\delta u=s^{-1} u$ and $\delta s^{-1} u=0$. Then the inclusion

$$
(N, d) \rightarrow(N, d) \oplus\left(A \otimes\left(U \oplus s^{-1} U\right), \delta\right)
$$

is both a cofibration and a quasi-isomorphism. Consider the isomorphism of $(A, d)-$ modules $\Phi:(N, d) \oplus\left(A \otimes\left(U \oplus s^{-1} U\right), \delta\right) \rightarrow(Q, D)$ defined by $\Phi(n)=v(n)-n$, $\Phi(u)=u$, and $\Phi\left(s^{-1} u\right)=d u+s^{-1} u$. Since $i$ is the restriction of $\Phi$ to $(N, d)$, it is both a cofibration and a quasi-isomorphism. Let $\pi:(Q, D) \rightarrow\left(M \otimes_{A} N, d\right)$ be the obvious projection. Then $\pi$ is a surjective morphism of $(A, d)$-modules and $\pi \circ i=v$.

Form the following commutative diagram of $(A, d)$-modules:

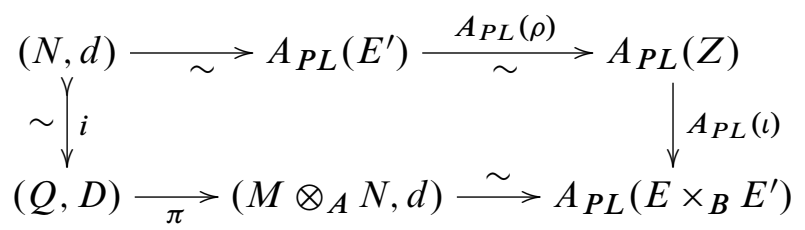

Since $A_{P L}(\iota)$ is surjective, there exists a lifting $\lambda:(Q, D) \rightarrow A_{P L}(Z)$ making the diagram commutative. Note that $\lambda$ is automatically a quasi-isomorphism. Consider the 
following commutative cube of $(A, d)$-modules:

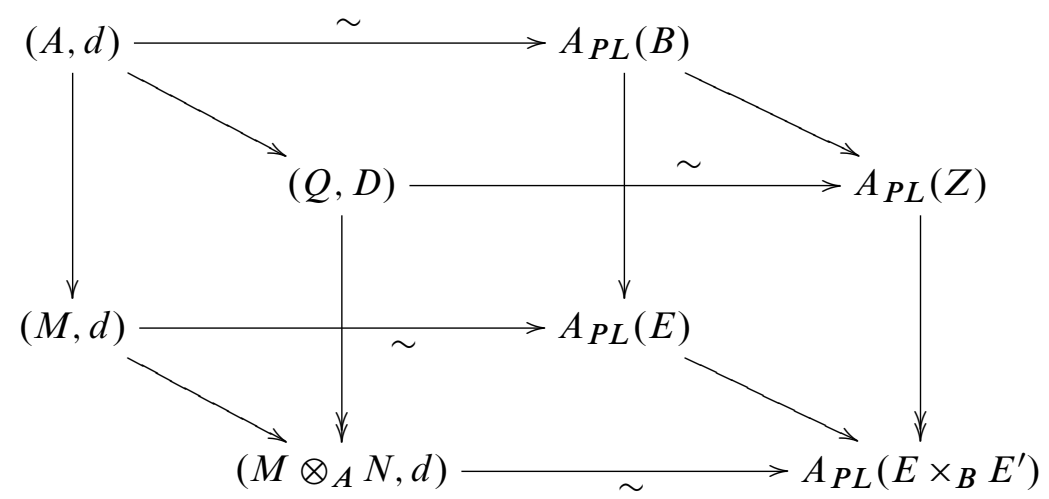

Form the pullback $(A, d)$-module $(J, D)=(M, d) \times\left(M \otimes_{A} N, d\right)$ ( $\left.Q, D\right)$ and the pullback cochain algebra $A_{P L}(E) \times_{A_{P L}\left(E \times_{B} E^{\prime}\right)} A_{P L}(Z)$. By the dual of the gluing lemma [1, II.1.2], [10, 8.13], the horizontal quasi-isomorphisms in the above cube induce a quasi-isomorphism of $(A, d)$-modules

$$
(J, D) \stackrel{\sim}{\longrightarrow} A_{P L}(E) \times_{A_{P L}}\left(E \times_{B} E^{\prime}\right) A_{P L}(Z) .
$$

By $[9,13.5]$, the canonical morphism

$$
A_{P L}\left(E *_{B} E^{\prime}\right) \rightarrow A_{P L}(E) \times_{A_{P L}\left(E \times_{B} E^{\prime}\right)} A_{P L}(Z)
$$

is a quasi-isomorphism and we obtain the following commutative diagram of $(A, d)-$ modules:

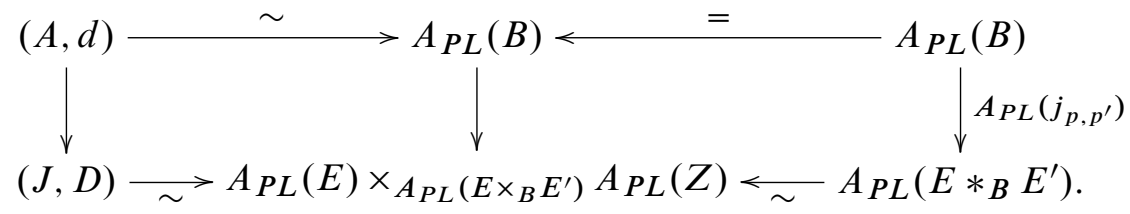

\section{Step 3: A quasi-isomorphism $(J, D) \stackrel{\sim}{\longrightarrow}(M, d) *_{(A, d)}(N, d)$}

We have

$$
(J, D)=\left(M \oplus N \oplus s^{-1} M \otimes_{A} N, D\right) .
$$

The action on $M \oplus N$ is the one of the direct sum, $a \cdot s^{-1} w=(-1)^{|a|} s^{-1} a w$, and the differential is given by $D m=d m+s^{-1} \gamma(m), D n=d n+s^{-1} v(n)$, and $D s^{-1} w=$ $-s^{-1} d w$. Here, $\gamma: M \rightarrow M \otimes_{A} N$ is the inclusion. Let $j:(A, d) \rightarrow(J, D)$ be the canonical morphism. If we write $a_{M}$ for the elements of $J$ which lie in the copy of $A$ coming from $M$ and $a_{N}$ for the elements of $J$ which lie in the copy of $A$ coming from $N$ then $j$ is given by $j(a)=a_{M}-a_{N}$. Since the join $(M, d) *(A, d)(N, d)$ is 
a semifree extension of $(A, d)$, by the lifting lemma [1, II.1.11], in order to finish the proof it is enough to construct a quasi-isomorphism of $(A, d)$-modules

$$
f:(J, D) \rightarrow(M, d) *{ }_{(A, d)}(N, d)=\left(A \oplus A \otimes s^{-1} X \otimes Y, d\right)
$$

such that the composite of $f \circ j$ is the inclusion $(A, d) \rightarrow(M, d) *_{(A, d)}(N, d)$. We define the map $f$ by $f\left(a_{M}\right)=\frac{1}{2} a, f(a \otimes x)=0, f\left(a_{N}\right)=-\frac{1}{2} a, f(a \otimes y)=0$, $f\left(s^{-1} a\right)=0, f\left(s^{-1} a \otimes x\right)=-\frac{1}{2}(-1)^{|a|} a d_{0} x, f\left(s^{-1} a \otimes y\right)=\frac{1}{2}(-1)^{|a|} a d_{0} y$, and $f\left(s^{-1} a \otimes x \otimes y\right)=(-1)^{|a|} a \otimes s^{-1} x \otimes y$. It is straightforward to check that $f$ is $A$-linear and obvious that $f \circ j$ is the inclusion. Consider an element $x \in X$ and write $d_{+} x=\sum_{i} a_{i} \otimes x_{i}$. As we have shown at the beginning of the proof of Proposition 3.2, $d d_{0} x=-\sum_{i}(-1)^{\left|a_{i}\right|} a_{i} d_{0} x_{i}$. Using this identity and a corresponding one for $y \in Y$, it is straightforward to check that $f$ commutes with the differentials. It remains to show that $f$ is a quasi-isomorphism. Consider the pushout $(A, d)-$ module $(R, d)=(A \oplus A \otimes X \oplus A \otimes Y, d)$ of the inclusions $(A, d) \rightarrow(M, d)$ and $(A, d) \rightarrow(N, d)$ and form the acyclic differential vector space

$$
\left(R \oplus s^{-1} R, D\right)=\left(A \oplus A \otimes X \oplus A \otimes Y \oplus s^{-1} A \oplus s^{-1} A \otimes X \oplus s^{-1} A \otimes Y, D\right)
$$

where $D r=d r+s^{-1} r$ and $D s^{-1} r=-s^{-1} d r$. Define a map $g: R \oplus s^{-1} R \rightarrow J$ by $g a=\frac{1}{2} a_{M}+\frac{1}{2} a_{N}, g(a \otimes x)=a \otimes x, g(a \otimes y)=a \otimes y, g\left(s^{-1} a\right)=s^{-1} a$,

$$
g\left(s^{-1} a \otimes x\right)=s^{-1} a \otimes x+\frac{1}{2}(-1)^{|a|}\left(a d_{0} x\right)_{M}-\frac{1}{2}(-1)^{|a|}\left(a d_{0} x\right)_{N},
$$

and

$$
g\left(s^{-1} a \otimes y\right)=s^{-1} a \otimes y-\frac{1}{2}(-1)^{|a|}\left(a d_{0} y\right)_{M}+\frac{1}{2}(-1)^{|a|}\left(a d_{0} y\right)_{N} .
$$

One easily checks that $f \circ g=0$. Write $A_{M}$ to denote the copy of $A$ in $J$ coming from $M$. Then

$$
J=A_{M} \oplus \operatorname{img} \oplus s^{-1} A \otimes X \otimes Y .
$$

Therefore $g$ is an isomorphism onto ker $f$. Using once more the identity $d d_{0} x=$ $-\sum_{i}(-1)^{\left|a_{i}\right|} a_{i} d_{0} x_{i}$, one checks that $g$ commutes with the differentials. Since $(R \oplus$ $\left.s^{-1} R, D\right)$ is acyclic, this implies that $f$ is a quasi-isomorphism. 


\section{The invariant Msecat}

Definition 5.1 Let $p: E \rightarrow B$ be fibration. We define Msecat $p$ to be the least integer $n$ such that there exists a commutative diagram of $A_{P L}(B)$-modules:

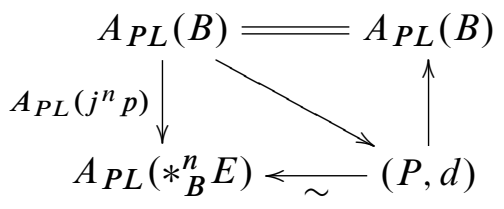

If no such $n$ exists we set Msecat $p=\infty$.

We first show that Msecat $p$ is a lower bound of secat $p$ which is closer to the sectional category than the classical lower bound nil ker $p^{*}$ :

Theorem 5.2 For any fibration $p: E \rightarrow B$, nil ker $p^{*} \leq$ Msecat $p$. If $B$ is normal then Msecat $p \leq$ secat $p$.

Proof Suppose that Msecat $p \leq n$. We show that nil ker $p^{*} \leq n$. Form the pullback:

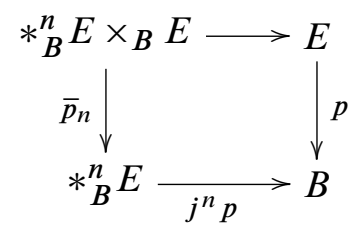

By Lemma 2.4, secat $\bar{p}_{n} \leq n$. Therefore nil ker $\bar{p}_{n}^{*} \leq n$. Since Msecat $p \leq n$, the join map $j^{n} p$ is injective in cohomology. Now consider elements $\alpha_{0}, \ldots, \alpha_{n} \in \operatorname{ker} p^{*}$. Since nil ker $\bar{p}_{n}^{*} \leq n$, we have $\left(j^{n} p\right)^{*}\left(\alpha_{0} \cup \cdots \cup \alpha_{n}\right)=0$. Since $\left(j^{n} p\right)^{*}$ is injective, $\alpha_{0} \cup \cdots \cup \alpha_{n}=0$. This shows that nil ker $p^{*} \leq n$.

Suppose now that $B$ is normal and that secat $p \leq n$. Then there exists a section $s: B \rightarrow *_{B}^{n} E$ of the join map $j^{n} p$. We therefore have the following commutative diagram of $A_{P L}(B)$-modules:

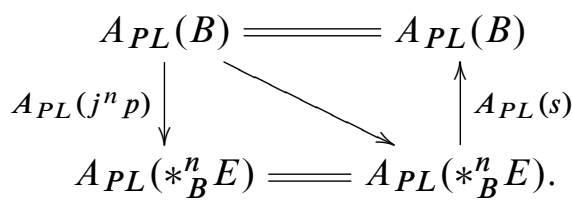

It follows that Msecat $p \leq n$.

Algebraic $8 \mathcal{G}$ Geometric Topology, Volume 6 (2006) 
The number Msecat $p$ can be calculated using the algebraic join construction of the previous sections. For the proof of this fact we need the following lemma:

Lemma 5.3 Let $p: E \rightarrow B$ be a fibration, $\alpha:(A, d) \stackrel{\sim}{\longrightarrow} A_{P L}(B)$ be a commutative cochain algebra model, $i:(A, d) \rightarrow(Q, d)$ be a cofibration of $(A, d)$-modules, and $\phi:(Q, d) \rightarrow A_{P L}\left(*_{B}^{n} E\right)$ be a morphism of $(A, d)$-modules such that $\phi \circ i=$ $A_{P L}\left(j^{n} p\right) \circ \alpha$. If Msecat $p \leq n$ then $i$ admits a retraction of $(A, d)$-modules.

Proof By definition, there is a commutative diagram of $A_{P L}(B)$-modules:

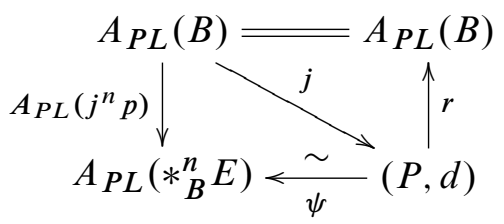

This is automatically a commutative diagram of $(A, d)$-modules. Form the following commutative diagram of $(A, d)$-modules:

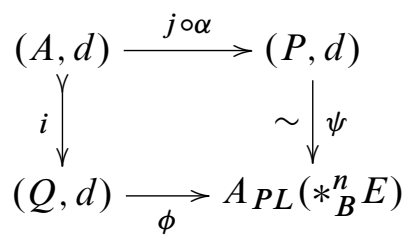

By the lifting lemma [1, II.1.11], there exists a morphism of $(A, d)$-modules $\lambda:(Q, d)$ $\rightarrow(P, d)$ such that $\lambda \circ i=j \circ \alpha$. We have obtained the following commutative diagram of $(A, d)$-modules:

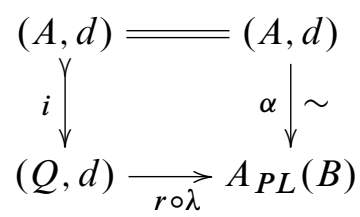

The lifting lemma [1, II.1.11] yields the required retraction of $i$.

Theorem 5.4 Let $p: E \rightarrow B$ be a fibration between simply connected spaces of finite type, $\alpha:(A, d) \stackrel{\sim}{\longrightarrow} A_{P L}(B)$ be a commutative cochain algebra model, and $(M, d)=(A \otimes(\mathbb{Q} \oplus X), d)$ be a semifree extension of $(A, d)$ such that there exists a quasi-isomorphism of $(A, d)$-modules $(M, d) \stackrel{\sim}{\longrightarrow} A_{P L}(E)$ extending $A_{P L}(p) \circ \alpha$. Then Msecat $p \leq n$ if and only if the inclusion $(A, d) \rightarrow *_{(A, d)}^{n}(M, d)$ admits a retraction of $(A, d)$-modules. 
Proof Suppose first that Msecat $p \leq n$. By Theorem 4.2, the morphism of $(A, d)$-modules $A_{P L}\left(j^{n} p\right) \circ \alpha$ extends to a quasi-isomorphism of $(A, d)$-modules $*_{(A, d)}^{n}(M, d) \stackrel{\sim}{\longrightarrow} A_{P L}\left(*_{B}^{n} E\right)$. By Lemma 5.3, the inclusion $(A, d) \rightarrow *_{(A, d)}^{n}(M, d)$ admits a retraction of $(A, d)$-modules.

Suppose now that the inclusion $(A, d) \rightarrow *_{(A, d)}^{n}(M, d)$ admits a retraction $\rho$ of $(A, d)-$ modules. Then the morphism of $A_{P L}(B)$-modules $A_{P L}(B) \otimes_{(A, d)} \rho$ is a retraction of the morphism of $A_{P L}(B)$-modules

$$
A_{P L}(B)=A_{P L}(B) \otimes_{(A, d)}(A, d) \rightarrow A_{P L}(B) \otimes_{(A, d)} *_{(A, d)}^{n}(M, d) .
$$

By Theorem 4.2, the morphism of $(A, d)$-modules $A_{P L}\left(j^{n} p\right) \circ \alpha$ extends to a quasiisomorphism of $(A, d)$-modules $\psi: *_{(A, d)}^{n}(M, d) \stackrel{\sim}{\longrightarrow} A_{P L}\left(*_{B}^{n} E\right)$. Consider the following commutative diagram of $A_{P L}(B)$-modules:

$$
\begin{gathered}
A_{P L}(B) \otimes_{A_{P L}(B)} A_{P L}(B) \frac{A_{P L}(B) \otimes_{\alpha} \alpha}{=} A_{P L}(B) \otimes_{(A, d)}(A, d) \\
\downarrow_{P L}(B) \otimes_{A_{P L}(B)} A_{P L}\left(j^{n} p\right) \\
A_{P L}(B) \otimes_{A_{P L}(B)} A_{P L}\left(*_{B}^{n} E\right) \underset{A_{P L}(B) \otimes_{\alpha} \psi}{\leftarrow} A_{P L}(B) \otimes_{(A, d)} *_{(A, d)}^{n}(M, d)
\end{gathered}
$$

Using $[9,6.10]$ one sees that $A_{P L}(B) \otimes_{\alpha} \psi$ is a quasi-isomorphism. The left hand vertical morphism is precisely $A_{P L}\left(j^{n} p\right)$. We have obtained the following commutative diagram of $A_{P L}(B)$-modules:

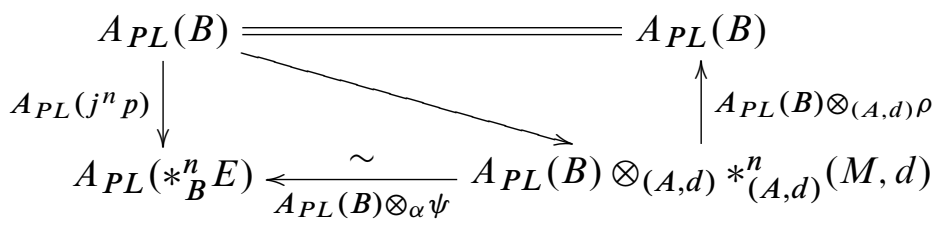

This shows that Msecat $p \leq n$.

Note that we have not yet shown that Msecat is a homotopy invariant. This is contained in the following proposition.

Proposition 5.5 Consider a commutative diagram

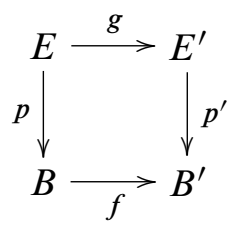

Algebraic 83 Geometric Topology, Volume 6 (2006) 
in which $p$ and $p^{\prime}$ are fibrations.

(a) If $f$ is a homotopy equivalence then Msecat $p^{\prime} \leq$ Msecat $p$.

(b) If $f$ and $g$ are homotopy equivalences then Msecat $p^{\prime}=$ Msecat $p$.

(c) If the diagram is a pullback and all spaces are simply connected and of finite type then Msecat $p \leq$ Msecat $p^{\prime}$.

Proof (a) Suppose that Msecat $p \leq n$. Choose a factorization $A_{P L}\left(j^{n} p^{\prime}\right)=\psi \circ i$ where $i: A_{P L}\left(B^{\prime}\right) \rightarrow(Q, d)$ is a cofibration of $A_{P L}\left(B^{\prime}\right)$-modules and $\psi:(Q, d) \rightarrow$ $A_{P L}\left(*_{B^{\prime}}^{n} E^{\prime}\right)$ is a quasi-isomorphism of $A_{P L}\left(B^{\prime}\right)$-modules. Then $A_{P L}\left(*_{f}^{n} g\right) \circ \psi \circ i=$ $A_{P L}\left(j^{n} p\right) \circ A_{P L}(f)$. By Lemma 5.3, $i$ admits a retraction of $A_{P L}\left(B^{\prime}\right)$-modules. This shows that Msecat $p^{\prime} \leq n$.

(b) This is a formal consequence of (a). Indeed, by (a), Msecat $p^{\prime} \leq$ Msecat $p$. But if $f$ and $g$ are homotopy equivalences then the homotopy inverses can be used to construct a commutative square

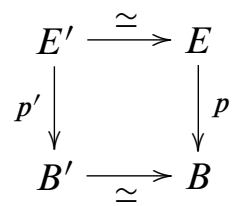

showing Msecat $p \leq$ Msecat $p^{\prime}$.

(c) Applying the functor $A_{P L}$ to the given square we obtain the following commutative diagram of commutative cochain algebras:

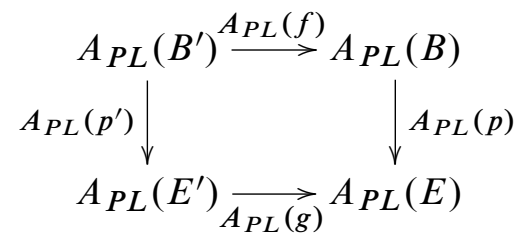

Let $\alpha:\left(A^{\prime}, d\right) \stackrel{\sim}{\longrightarrow} A_{P L}\left(B^{\prime}\right)$ be a Sullivan model. Choose factorizations $A_{P L}(f) \circ \alpha=$ $\psi \circ i$ and $A_{P L}\left(p^{\prime}\right) \circ \alpha=\phi \circ j$ such that $i:\left(A^{\prime}, d\right) \rightarrow(A, d)$ and $j:\left(A^{\prime}, d\right) \rightarrow$ $\left(M^{\prime}, d\right)$ are inclusions of relative Sullivan algebras and $\psi:(A, d) \rightarrow A_{P L}(B)$ and $\phi:\left(M^{\prime}, d\right) \rightarrow A_{P L}\left(E^{\prime}\right)$ are quasi-isomorphisms. Then, by $[9,15(\mathrm{c})]$, the induced morphism of cochain algebras

$$
(M, d)=(A, d) \otimes_{\left(A^{\prime}, d\right)}\left(M^{\prime}, d\right) \rightarrow A_{P L}(E)
$$

is a quasi-isomorphism. Note that $\left(M^{\prime}, d\right)$ is a semifree extension of $\left(A^{\prime}, d\right)$ and $(M, d)$ is a semifree extension of $(A, d)$. Suppose that Msecat $p^{\prime} \leq n$. By the 
preceding theorem, the inclusion $\left(A^{\prime}, d\right) \rightarrow *_{\left(A^{\prime}, d\right)}^{n}\left(M^{\prime}, d\right)$ admits a retraction $\rho$ of $\left(A^{\prime}, d\right)$-modules. As remarked in Remarks 3.4(iii),

$$
*_{(A, d)}^{n}(M, d)=(A, d) \otimes_{\left(A^{\prime}, d\right)} *_{\left(A^{\prime}, d\right)}^{n}\left(M^{\prime}, d\right) .
$$

The morphism of $(A, d)$-modules

$$
A \otimes_{A^{\prime}} \rho:(A, d) \otimes_{\left(A^{\prime}, d\right)} *_{\left(A^{\prime}, d\right)}^{n}\left(M^{\prime}, d\right) \rightarrow(A, d) \otimes_{\left(A^{\prime}, d\right)}\left(A^{\prime}, d\right)=(A, d)
$$

is a retraction of the inclusion $(A, d) \rightarrow *_{(A, d)}^{n}(M, d)$. By the preceding theorem, this implies that Msecat $p \leq n$.

The next proposition shows that the invariant Msecat is a generalization of the wellknown invariant Mcat of spaces. Let $B$ be a simply connected space of finite type with Sullivan model $(\Lambda V, d)$. By definition, Mcat $B$ is the least integer $n$ such that for some (equivalently: any) Sullivan model $(\Lambda V \otimes \Lambda W, d) \stackrel{\sim}{\longrightarrow}\left(\Lambda V / \Lambda^{>n} V, d\right)$ of the projection $(\Lambda V, d) \rightarrow\left(\Lambda V / \Lambda^{>n} V, d\right)$, the inclusion $(\Lambda V, d) \rightarrow(\Lambda V \otimes \Lambda W, d)$ admits a retraction of $(\Lambda V, d)$-modules. If no such $n$ exists, Mcat $B=\infty$.

Proposition 5.6 Let $B$ be a simply connected pointed space of finite type. Consider the evaluation fibration $e v_{1}: P B \rightarrow B, \omega \mapsto \omega(1)$. Then Msecat $e v_{1}=$ Mcat $B$.

Proof Let $\alpha:(\Lambda V, d) \stackrel{\sim}{\longrightarrow} A_{P L}(B)$ be a Sullivan model of $B$. Denote the projection $(\Lambda V, d) \rightarrow\left(\Lambda V / \Lambda^{>n} V, d\right)$ by $q_{n}$ and choose a factorization $q_{n}=\phi \circ i$ where $i:(\Lambda V, d) \rightarrow(\Lambda V \otimes \Lambda W, d)$ is the inclusion of a relative Sullivan algebra and $\phi:(\Lambda V \otimes \Lambda W, d) \rightarrow\left(\Lambda V / \Lambda^{>n} V, d\right)$ is a quasi-isomorphism. Choose a factorization $A_{P L}\left(j^{n} e v_{1}\right) \circ \alpha=\psi \circ j$ where $j:(\Lambda V, d) \rightarrow(\Lambda V \otimes \Lambda X, d)$ is the inclusion of a relative Sullivan algebra and $\psi:(\Lambda V \otimes \Lambda X, d) \rightarrow A_{P L}\left(*_{B}^{n} P B\right)$ is a quasi-isomorphism. It follows from Félix and Halperin [6] that there exist morphisms of commutative cochain algebras $\sigma:(\Lambda V \otimes \Lambda X, d) \rightarrow(\Lambda V \otimes \Lambda W, d)$ and $\rho:(\Lambda V \otimes \Lambda W, d) \rightarrow(\Lambda V \otimes \Lambda X, d)$ such that $\sigma \circ j=i$ and $\rho \circ i=j$. This implies that Mcat $B \leq n$ if and only if $j$ admits a retraction of $(\Lambda V, d)$-modules. Let $(M, d)$ be a semifree extension of $(\Lambda V, d)$ such that there exists a quasi-isomorphism of $(\Lambda V, d)$-modules $(M, d) \stackrel{\sim}{\longrightarrow} A_{P L}(P B)$ which extends $A_{P L}\left(e v_{1}\right) \circ \alpha$. By Theorem 4.2, the morphism of $(\Lambda V, d)$-modules $A_{P L}\left(j^{n} e v_{1}\right) \circ \alpha$ extends to a quasi-isomorphism of $(\Lambda V, d)-$ modules $*_{(\Lambda V, d)}^{n}(M, d) \stackrel{\sim}{\longrightarrow} A_{P L}\left(*_{B}^{n} P B\right)$. Use the lifting lemma [1, II.1.11] to construct quasi-isomorphisms of $(\Lambda V, d)$-modules $\beta: *_{(\Lambda V, d)}^{n}(M, d) \stackrel{\sim}{\longrightarrow}(\Lambda V \otimes \Lambda X, d)$ and $\gamma:(\Lambda V \otimes \Lambda X, d) \stackrel{\sim}{\longrightarrow} *_{(\Lambda V, d)}^{n}(M, d)$ such that $\beta$ extends $j$ and $\gamma \circ j$ is the inclusion of $(\Lambda V, d)$. We obtain that Mcat $B \leq n$ if and only if the inclusion $(\Lambda V, d) \rightarrow *_{(\Lambda V, d)}^{n}(M, d)$ admits a retraction of $(\Lambda V, d)$-modules. By Theorem 5.4, this is the case if and only if Msecate $e v_{1} \leq n$. 
Recall that an upper bound for the sectional category of a surjective fibration is given by the Lusternik-Schnirelmann category of the base space. The following is the analogous result for Msecat and Mcat.

Proposition 5.7 Let $B$ be a simply connected space of finite type. For any surjective fibration $p: E \rightarrow B$, Msecat $p \leq$ Mcat $B$.

Proof Recall that for us simply connected spaces are non-empty. Fix any base point in $B$ and consider the evaluation fibration $e v_{1}: P B \rightarrow B$. Since $p$ is surjective, $E \neq \varnothing$. Since $P B$ is contractible, there exists a continuous map $\lambda: P B \rightarrow E$ such that $p \circ \lambda=$ $e v_{1}$. By Proposition 5.5(a) and Proposition 5.6, Msecat $p \leq$ Msecate $e v_{1}=$ Mcat $B$.

Remark 5.8 K Hess [11] has shown that the invariant Mcat coincides for simply connected spaces with rational Lusternik-Schnirelmann category. This result does not generalize to sectional category. Indeed, D Stanley [17] has constructed a fibration $p$ with fiber $S^{2}$ whose rational sectional category is 1 . By Vandembroucq [18], any such fibration satisfies Msecat $p=0$.

\section{Topological complexity}

In [3] and [4], M Farber defined the topological complexity of a space $X, \mathrm{TC}(X)$, to be the sectional category of the evaluation fibration $e v_{0,1}: X^{I} \rightarrow X \times X, \omega \mapsto(\omega(0), \omega(1))$. This invariant has proved to be very useful in the study of the motion planning problem in robotics. Note that Farber's definition of TC differs by 1 from the one given here. In this section we study the invariant

$$
\operatorname{MTC}(X)=\operatorname{Msecat}\left(e v_{0,1}: X^{I} \rightarrow X \times X\right) .
$$

In order to simplify the presentation we restrict our attention to simply connected spaces of finite type having the homotopy type of $\mathrm{CW}$ complexes.

The evaluation fibration $e v_{0,1}: X^{I} \rightarrow X \times X$ is the mapping path fibration associated to the diagonal map $\Delta: X \rightarrow X \times X$. We may therefore identify the map $e v_{0,1}^{*}: H^{*}(X \times$ $X) \rightarrow H^{*}\left(X^{I}\right)$ with the cup product $\cup: H^{*}(X) \otimes H^{*}(X) \rightarrow H^{*}(X)$.

Proposition 6.1 We have nil $\operatorname{ker} \cup \leq \operatorname{MTC}(X) \leq \mathrm{TC}(X)$ and Mcat $X \leq \operatorname{MTC}(X)$ $\leq 2$ Mcat $X$.

Proof The first inequalities follow from Theorem 5.2. By Proposition 5.7 and $[9$, 30.2], MTC $(X) \leq \operatorname{Mcat}(X \times X)=2 \operatorname{Mcat} X$. For the remaining inequality consider 
the map $f: X \rightarrow X \times X, x \mapsto(*, x)$ where $* \in X$ is any base point and form the following pullback diagram:

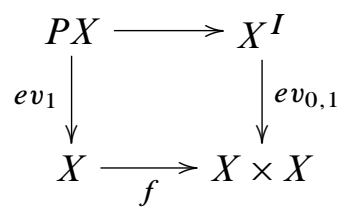

By Proposition 5.6 and Proposition 5.5(c), Mcat $X=$ Msecat $e v_{1} \leq$ Msecate $e v_{0,1}=$ $\operatorname{MTC}(X)$.

Consider a space $X$ with Sullivan model $(\Lambda V, d)$. A Sullivan model of the product space $X \times X$ is then given by $\left(\Lambda\left(V \oplus V^{\prime}\right), d\right)=(\Lambda V, d) \otimes\left(\Lambda V^{\prime}, d\right)$ where $\left(\Lambda V^{\prime}, d\right)$ is second copy of $(\Lambda V, d)$. As is shown in [9, pages 206-207], a model of the evaluation fibration (and the diagonal map) is given by the inclusion

$$
\left(\Lambda\left(V \oplus V^{\prime}\right), d\right) \rightarrow\left(\Lambda\left(V \oplus V^{\prime}\right) \otimes \Lambda \bar{V}, d\right)
$$

where

$$
d(\bar{v})=v^{\prime}-v-\sum_{i=1}^{\infty} \frac{(\zeta d)^{i}}{i !}(v) .
$$

Here, $\zeta$ is the derivation of degree -1 defined by $\zeta(v)=\zeta\left(v^{\prime}\right)=\bar{v}$ and $\zeta(\bar{v})=0$. Using this explicit semifree extension of $\left(\Lambda\left(V \oplus V^{\prime}\right), d\right)$ and the formula for the differential of the iterated join given in Remarks 3.4(ii), one can calculate the invariant $\operatorname{MTC}(X)$ from $(\Lambda V, d)$. We remark that $d_{0} \bar{v}=v^{\prime}-v$ and that $d_{0} x=0$ for $x \in \Lambda^{>1} \bar{V}$. Note also that if $(\Lambda V, d)$ is the minimal Sullivan model of $X$ then $\left(\Lambda\left(V \oplus V^{\prime}\right) \otimes \Lambda \bar{V}, d\right)$ is a minimal semifree $\left(\Lambda\left(V \oplus V^{\prime}\right), d\right)$-module.

The following proposition provides an upper bound for MTC.

Proposition 6.2 Let $(A, d)$ be a commutative cochain algebra model of $X$ with multiplication $\mu$. Then $\operatorname{MTC}(X) \leq \operatorname{nil} \operatorname{ker} \mu$.

Proof Suppose that nil ker $\mu \leq n$. We show that the inclusion

$$
i:\left(\Lambda\left(V \oplus V^{\prime}\right), d\right) \rightarrow *_{\left(\Lambda\left(V \oplus V^{\prime}\right), d\right)}^{n}\left(\Lambda\left(V \oplus V^{\prime}\right) \otimes \Lambda \bar{V}, d\right)
$$

admits a retraction of $\left(\Lambda\left(V \oplus V^{\prime}\right), d\right)$-modules. Choose a quasi-isomorphism of commutative cochain algebras $\alpha:(\Lambda V, d) \stackrel{\sim}{\longrightarrow}(A, d)$. Consider the tensor product algebra $(A, d) \otimes(A, d)=(A \otimes A, d)$ and the $(A \otimes A, d)$-semifree extension of $(A \otimes A, d)$ defined by

$$
(M, d)=(A \otimes A, d) \otimes_{\left(\Lambda\left(V \oplus V^{\prime}\right), d\right)}\left(\Lambda\left(V \oplus V^{\prime}\right) \otimes \Lambda \bar{V}, d\right)=(A \otimes A \otimes \Lambda \bar{V}, d) .
$$


We have

$$
*_{(A \otimes A, d)}^{n}(M, d)=\left(A \otimes A \oplus A \otimes A \otimes s^{-n}\left(\Lambda^{+} \bar{V}\right)^{\otimes n+1}, d\right) .
$$

Consider an element $s^{-n} x_{0} \otimes \cdots \otimes x_{n} \in s^{-n}\left(\Lambda^{+} \bar{V}\right)^{\otimes n+1}$. If one of the $x_{i}$ lies in $\Lambda^{>1} \bar{V}$ then $d\left(s^{-n} x_{0} \otimes \cdots \otimes x_{n}\right)$ has no term in $A \otimes A$. Since nil ker $\mu \leq n$, this also holds if all $x_{i} \in \bar{V}$. We can thus define an $(A \otimes A, d)$-module retraction $r$ of the inclusion $j:(A \otimes A, d) \rightarrow *_{(A \otimes A, d)}^{n}(M, d)$ by sending $A \otimes A \otimes s^{-n}\left(\Lambda^{+} \bar{V}\right)^{\otimes n+1}$ to 0 . By Remarks 3.4(iii), the map $j$ is obtained by applying the functor $(A \otimes$ $A, d) \otimes_{\left(\Lambda\left(V \oplus V^{\prime}\right), d\right)}$ - to the inclusion $i$. Consider the following commutative diagram of $\left(\Lambda\left(V \oplus V^{\prime}\right), d\right)$-modules:

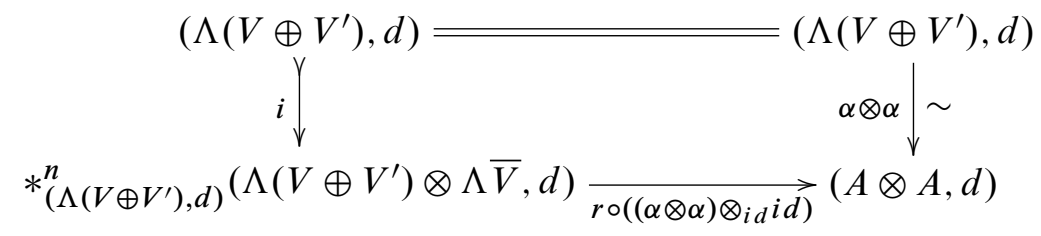

The lifting lemma [1, II.1.11] yields the required retraction of $i$.

Note that the number nil ker $\mu$ is in general not the same for different commutative cochain algebra models of $X$. Given a commutative graded algebra $A$ with multiplication $\mu$, the number nil ker $\mu$ can be determined using the following lemma:

Lemma 6.3 Let $\Lambda W$ be a commutative graded algebra and $I \subset \Lambda W$ be an ideal such that $A=\Lambda W / I$. Let $\Lambda W^{\prime}$ be a second copy of $\Lambda W$ and $J \subset \Lambda\left(W \oplus W^{\prime}\right)=\Lambda W \otimes$ $\Lambda W^{\prime}$ be the ideal $I \otimes \Lambda W^{\prime}+\Lambda W \otimes I^{\prime}$ where $I^{\prime}$ is the ideal of $\Lambda W^{\prime}$ corresponding to $I$. Let finally $\mathcal{B}$ be a basis of the graded vector space $W$. Then nil $\operatorname{ker} \mu \leq n$ if and only if, for all $w_{0}, \ldots, w_{n} \in \mathcal{B},\left(w_{0}^{\prime}-w_{0}\right) \cdots\left(w_{n}^{\prime}-w_{n}\right) \equiv 0 \bmod J$.

Proof Denote the multiplication $\Lambda\left(W \oplus W^{\prime}\right)=\Lambda W \otimes \Lambda W^{\prime} \rightarrow \Lambda W$ by $m$. We have $A \otimes A=\Lambda\left(W \oplus W^{\prime}\right) / J$ and $\operatorname{ker} \mu=\operatorname{ker} m /(J \cap \operatorname{ker} m)$. It suffices to show that ker $m$ is the ideal of $\Lambda\left(W \oplus W^{\prime}\right)$ generated by the elements $w^{\prime}-w, w \in \mathcal{B}$. Denote this ideal by $K$. Obviously, $K \subset \operatorname{ker} m$. Note also that $K \cap \Lambda W=0$. In order to show the equality $K=\operatorname{ker} m$ we show that $\Lambda W \oplus K=\Lambda\left(W \oplus W^{\prime}\right)$. For this it is enough to show that for each $n \geq 1, \Lambda W \otimes \Lambda^{n} W^{\prime} \subset \Lambda W \oplus K$. We proceed by induction. Consider $w \in \mathcal{B}$ and $\xi \in \Lambda W$. We have $\xi w^{\prime}=\xi w+\xi\left(w^{\prime}-w\right) \in \Lambda W \oplus K$. Suppose the assertion holds for some $n \geq 1$. Consider $\zeta \in \Lambda W \otimes \Lambda^{n} W^{\prime}$ and $w \in \mathcal{B}$. By the inductive hypothesis, $\zeta \in \Lambda W \oplus K$. Write $\zeta=\theta+k$ with $\theta \in \Lambda W$ and $k \in K$. By the inductive hypothesis, $\theta w^{\prime} \in \Lambda W \oplus K$. Since $K$ is an ideal, $k w^{\prime} \in K$. It follows that $\zeta w^{\prime} \in \Lambda W \oplus K$. This closes the induction and the result follows. 
Recall that a space $X$ is called formal if $H^{*}(X)$ is a commutative cochain algebra model of $X$. Proposition 6.2 immediately implies the following:

Proposition 6.4 If $X$ is formal then $\operatorname{MTC}(X)=$ nil $\operatorname{ker} \cup$.

Example 6.5 The simplest example of a non-formal space is the space

$$
X=S_{a}^{3} \vee S_{b}^{3} \cup e^{8} \cup e^{8}
$$

where the 8-cells are attached by means of the iterated Whitehead products $\left[S_{a}^{3},\left[S_{a}^{3}, S_{b}^{3}\right]\right]$ and $\left[S_{b}^{3},\left[S_{a}^{3}, S_{b}^{3}\right]\right]$. We show that this space satisfies $\operatorname{MTC}(X)=3$ and nil ker $\cup=2$. For degree reasons, the space $X$ has the same cohomology algebra as the wedge of spheres $S^{3} \vee S^{3} \vee S^{8} \vee S^{8}$. Therefore $X$ satisfies nil ker $\cup=2$. Indeed, since $\mathrm{TC} \leq 2 \mathrm{cat}$, the topological complexity of a wedge of spheres is $\leq 2$. On the other hand, any space with at least two cohomology generators satisfies nil ker $\cup \geq 2$ : if $\xi$ and $\theta$ are two cohomology generators then $(\xi \otimes 1-1 \otimes \xi)(\theta \otimes 1-1 \otimes \theta)$ is a nontrivial product in $\operatorname{ker} \cup$. The minimal Sullivan model of $X$ is the algebra $(\Lambda(V), d)$ where the graded $\mathbb{Q}$-vector space $V$ is generated by cocycles $a$ and $b$ of degree 3 , an element $u$ of degree 5 with $d u=a b$, and elements of degree $>8$. Consider the $d$-stable ideal $I=(\Lambda V)^{\geq 9}$ and form the quotient algebra $(A, d)=(\Lambda V / I, d)$. Since $(I, d)$ is acyclic, the projection $(\Lambda V, d) \rightarrow(\Lambda V / I, d)$ is a quasi-isomorphism. Consider the ideal $J=I \otimes \Lambda V^{\prime}+\Lambda V \otimes I^{\prime} \subset \Lambda\left(V \oplus V^{\prime}\right)$ as in Lemma 6.3. We have $\left(a^{\prime}-a\right)\left(b^{\prime}-b\right)\left(u^{\prime}-u\right) \not \equiv 0 \bmod J$. Since $a, b, u$ are of odd degree, any longer nonzero product of the form $\left(v_{0}^{\prime}-v_{0}\right) \cdots\left(v_{n}^{\prime}-v_{n}\right)$ must contain at least one factor $v_{i}^{\prime}-v_{i}$ with $\left|v_{i}\right| \geq 9$. For $n \geq 3$ any such product is therefore an element of $J$. By Proposition 6.2 and Lemma 6.3, this implies that $\operatorname{MTC}(X) \leq 3$. We show that $\operatorname{MTC}(X)>2$. The differential of the generators $\bar{a}, \bar{b}$, and $\bar{u}$ of the model $\left(\Lambda\left(V \oplus V^{\prime}\right) \otimes \Lambda \bar{V}, d\right)$ of $X^{I}$ is given by $d \bar{a}=a^{\prime}-a, d \bar{b}=b^{\prime}-b$, and $d \bar{u}=u^{\prime}-u+\alpha \otimes \bar{a}+\beta \otimes \bar{b}$ where $\alpha, \beta \in \Lambda\left(V \oplus V^{\prime}\right)$ are some elements of degree 3. A straightforward calculation shows that $\alpha=-\frac{1}{2}\left(b+b^{\prime}\right)$ and $\beta=\frac{1}{2}\left(a+a^{\prime}\right)$. This information is, however, not needed for the calculations. It suffices to show that the inclusion

$$
\begin{aligned}
\left(\Lambda\left(V \oplus V^{\prime}\right), d\right) & \rightarrow *_{\left(\Lambda\left(V \oplus V^{\prime}\right), d\right)}^{2}\left(\Lambda\left(V \oplus V^{\prime}\right) \otimes \Lambda \bar{V}, d\right) \\
& =\left(\Lambda\left(V \oplus V^{\prime}\right) \oplus \Lambda\left(V \oplus V^{\prime}\right) \otimes s^{-2} \Lambda^{+} \bar{V} \otimes \Lambda^{+} \bar{V} \otimes \Lambda^{+} \bar{V}, d\right)
\end{aligned}
$$

is not injective in cohomology. The element $z=\left(a^{\prime}-a\right)\left(b^{\prime}-b\right)\left(u^{\prime}-u\right)$ is a cocycle of degree 11 in $\Lambda\left(V \oplus V^{\prime}\right)$ which is not a coboundary. In the 2-fold join, however, we have

$d\left(s^{-2}(\bar{a} \otimes \bar{b} \otimes \bar{u}+\bar{b} \otimes \bar{u} \otimes \bar{a}+\bar{u} \otimes \bar{a} \otimes \bar{b}-\bar{a} \otimes \bar{u} \otimes \bar{b}-\bar{b} \otimes \bar{a} \otimes \bar{u}-\bar{u} \otimes \bar{b} \otimes \bar{a})\right)=-6 z$ so that $[z]=0 \in H^{11}\left(*_{\left(\Lambda\left(V \oplus V^{\prime}\right), d\right)}^{2}\left(\Lambda\left(V \oplus V^{\prime}\right) \otimes \Lambda \bar{V}, d\right)\right)$. 
Our last result is the fact that the difference $\operatorname{MTC}(X)$ - nil ker $\cup$ can be arbitrarily large:

Proposition 6.6 (i) For any $n \in \mathbb{N}$ there exists a finite $C W$-complex $X$ such that $\operatorname{MTC}(X)-$ nil ker $\cup \geq n$.

(ii) There exists a space $X$ such that $\operatorname{MTC}(X)=\infty$ and nil $\operatorname{ker} \cup<\infty$.

Proof (i) Let $Z$ be a simply connected finite CW-complex having the same cohomology algebra as a wedge of spheres $Y$ and satisfying Mcat $Z=3$. Such a space has for instance been constructed by Kahl and Vandembroucq [14]. Let $X$ be the $n$-fold product of the space $Z, X=Z^{n}$. Then $X$ is a finite CW-complex which has the same cohomology algebra as $Y^{n}$ and satisfies nil $\operatorname{ker} \cup \leq \mathrm{TC}\left(Y^{n}\right) \leq$ $2 \operatorname{cat}\left(Y^{n}\right) \leq 2 n$ cat $Y=2 n$. On the other hand, by Proposition 6.1 and $[9,30.2]$, $\operatorname{MTC}(X) \geq \operatorname{Mcat}(X)=n \operatorname{Mcat}(Z)=3 n$.

(ii) It suffices to take a space $X$ such that Mcat $X=\infty$ and nil $H^{+}(X)<\infty$. Such a space has been constructed by Félix, Halperin and Thomas [7].

\section{References}

[1] HJ Baues, Algebraic homotopy, Cambridge Studies in Advanced Mathematics 15, Cambridge University Press, Cambridge (1989) MR985099

[2] O Cornea, G Lupton, J Oprea, D Tanré, Lusternik-Schnirelmann category, Mathematical Surveys and Monographs 103, American Mathematical Society, Providence, RI (2003) MR1990857

[3] M Farber, Topological complexity of motion planning, Discrete Comput. Geom. 29 (2003) 211-221 MR1957228

[4] M Farber, Instabilities of robot motion, Topology Appl. 140 (2004) 245-266 MR2074919

[5] A Fassò Velenik, Relative homotopy invariants of the type of the LusternikSchnirelmann category, PhD thesis, FU Berlin (2002)

[6] Y Félix, S Halperin, Rational LS category and its applications, Trans. Amer. Math. Soc. 273 (1982) 1-38 MR664027

[7] Y Félix, S Halperin, J-C Thomas, L.S. catégorie et suite spectrale de Milnor-Moore (une nuit dans le train), Bull. Soc. Math. France 111 (1983) 89-96 MR710377

[8] Y Félix, S Halperin, J-C Thomas, Differential graded algebras in topology, from: "Handbook of algebraic topology", North-Holland, Amsterdam (1995) 829-865 MR1361901 
[9] Y Félix, S Halperin, J-C Thomas, Rational homotopy theory, Graduate Texts in Mathematics 205, Springer, New York (2001) MR1802847

[10] P G Goerss, J F Jardine, Simplicial homotopy theory, Progress in Mathematics 174, Birkhäuser Verlag, Basel (1999) MR1711612

[11] K P Hess, A proof of Ganea's conjecture for rational spaces, Topology 30 (1991) 205-214 MR1098914

[12] I M James, On category, in the sense of Lusternik-Schnirelmann, Topology 17 (1978) 331-348 MR516214

[13] I M James, Lusternik-Schnirelmann category, from: "Handbook of algebraic topology", North-Holland, Amsterdam (1995) 1293-1310 MR1361912

[14] T Kahl, L Vandembroucq, Gaps in the Milnor-Moore spectral sequence, Bull. Belg. Math. Soc. Simon Stevin 9 (2002) 265-277 MR2017081

[15] A Schwarz, The genus of a fiber space, Amer. Math. Soc. Transl. 55 (1966) 49-140

[16] S Smale, On the topology of algorithms. I, J. Complexity 3 (1987) 81-89 MR907191

[17] D Stanley, The sectional category of spherical fibrations, Proc. Amer. Math. Soc. 128 (2000) 3137-3143 MR1691006

[18] L Vandembroucq, Sur le transfert de Becker et Gottlieb, Bull. Belg. Math. Soc. Simon Stevin 6 (1999) 605-614 MR1732893

L Fernández Suárez, T Kahl, L Vandembroucq:

Universidade do Minho, Centro de Matemática, Campus de Gualtar

4710-057 Braga, Portugal

P Ghienne:

Université d'Artois, Faculté des Sciences Jean Perrin

Laboratoire de Mathématiques, Rue Jean Souvraz - SP 18

62307 Lens Cedex, France

lfernandez@math.uminho.pt, ghienne@euler.univ-artois.fr, kahl@math.uminho.pt, lucile@math.uminho.pt

Received: 1 November 2005 Revised: 11 January 2006 